P. J. Trunk, I. Boxx, C. Heeger, W. Meier, B. Böhm, A. Dreizler

Premixed flame propagation in turbulent flow by means of stereoscopic PIV and dual-plane OH-PLIF at sustained $\mathrm{kHz}$ repetition rates

Published in the Proceedings of the Combustion Institute, 2012.

Volume 34, Issue 2, 2013, Pages 3565-3572

Original publication available at

http://www.sciencedirect.com/science/article/pii/S1540748912001332

http://dx.doi.org/10.1016/j.proci.2012.06.025 


\title{
Premixed Flame Propagation in Turbulent Flow by means of Stereoscopic PIV and Dual-Plane OH-PLIF at sustained $\mathrm{KHz}$ Repetition Rates
}

\author{
P. J. Trunk ${ }^{1}$, I. Boxx ${ }^{2}$, C. Heeger ${ }^{1}$, W. Meier $^{2}$, B. Böhm ${ }^{3 *}$, A. Dreizler ${ }^{1}$ \\ ${ }^{1}$ : Fachgebiet Reaktive Strömungen und Messtechnik (RSM), Center of Smart Interfaces (CSI) \\ Technische Universität Darmstadt \\ Petersenstrasse 32, D - 64287 Darmstadt, Germany \\ ${ }^{2}$ : Deutsches Zentrum für Luft- und Raumfahrt (DLR), Institut für Verbrennungstechnik \\ Pfaffenwaldring 38, D - 70569 Stuttgart, Germany \\ ${ }^{3}$ : Fachgebiet Energie- und Kraftwerkstechnik (EKT), Technische Universität Darmstadt \\ Petersenstrasse 30, D - 64287 Darmstadt, Germany
}

*Corresponding author: bboehm@ekt.tu-darmstadt.de

Benjamin Böhm

Fachgebiet Energie- und Kraftwerkstechnik, Technische Universität Darmstadt

Petersenstrasse 30

D - 64287 Darmstadt

Germany

Fax: +49 6151166555

Colloquium:

Diagnostics,

(Turbulent Flames) 


\section{Word Count According to Method 1}

Overall: 6112 words

\section{Details:}

Text: Count by Microsoft Word: 3306 words

Equations: $4 \times(1$ line +2 blank lines $) \times(1$ column $)(7.6$ words/line/column $)=91$ words

References: $((22+2)$ references $) \times(2.3$ lines/reference $) \times(7.6$ words/line $)=419$ words

Figures and Captions: (According to the following list) $2091+205$ words

Fig. 1: $(45 \mathrm{~mm}+10 \mathrm{~mm}) \times(1$ columns $) \times(2.2$ words $/ \mathrm{mm} /$ column $)=121$ words

Fig. 2: $(45 \mathrm{~mm}+10 \mathrm{~mm}) \times(1$ columns $) \times(2.2$ words $/ \mathrm{mm} /$ column $)=121$ words

Fig. 3: $(37 \mathrm{~mm}+10 \mathrm{~mm}) \times(1$ columns $) \times(2.2$ words $/ \mathrm{mm} /$ column $)=103$ words

Fig. 4: $(55 \mathrm{~mm}+10 \mathrm{~mm}) \times(1$ columns $) \times(2.2$ words $/ \mathrm{mm} /$ column $)=143$ words

Fig. 5: $(63 \mathrm{~mm}+10 \mathrm{~mm}) \times(1$ columns $) \times(2.2$ words $/ \mathrm{mm} /$ column $)=161$ words

Fig. 6: $(97 \mathrm{~mm}+10 \mathrm{~mm}) \times(2$ columns $) \times(2.2$ words $/ \mathrm{mm} /$ column $)=470$ words

Fig. 7: $(161 \mathrm{~mm}+10 \mathrm{~mm}) \times(2$ column $) \times(2.2$ words $/ \mathrm{mm} /$ column $)=814$ words

Fig. 8: $(62 \mathrm{~mm}+10 \mathrm{~mm}) \times(1$ columns $) \times(2.2$ words $/ \mathrm{mm} /$ column $)=158$ words

Figure Captions: Count by Microsoft Word (last page): 205 words

\section{Colloquium}

Diagnostics

\section{Colored Figures}

Herewith we affirm to pay color reproduction charges for 5 figures (this includes figures $3,4,5,6,7)$. 


\begin{abstract}
This paper describes an experimental study on the propagation of premixed flames through a flow with well-defined turbulence characteristics. In this study, multi-plane OH planar laserinduced fluorescence (OH-PLIF) and stereoscopic particle image velocimetry (SPIV) were applied simultaneously at $10 \mathrm{kHz}$ to measure the local three-dimensional displacement velocity of unstabilized, freely propagating flames passing through a premixed flow of methane and air. The dual-plane OH-PLIF data was used to track both reaction zone location and flame-normal orientation, while SPIV was used to track the three-component velocity field. The vectorial difference of the two produces a direct, time-resolved measurement of the local 3D displacement velocity of the flame. The use of unstabilized, freely propagating flames eliminates spatiotemporal dependence of local 3D displacement velocity associated with burner- or aerodynamically-stabilized test flames. Statistics of local 3D displacement velocities are presented. These show values ranging from slightly negative up to approximately eight times the laminar flame speed. Instantaneous flame-normal orientation with respect to the direction of local fluid velocity is identified as one parameter affecting instantaneous displacement velocities.
\end{abstract}

\title{
Keywords
}

High-speed imaging, premixed combustion, flame propagation, flame speed, turbulent combustion 


\section{Introduction}

The wrinkled laminar flamelet has proven an especially useful concept in the development of reduced-order models for the numerical simulation of turbulent flames [1]. This concept is associated with a combustion regime wherein heat-release reactions occur in thin layers throughout a turbulent flow. These layers, or "flamelets", are believed to retain the properties of premixed laminar flames. The primary effect of turbulence in this regime is believed to be the wrinkling and stretching of these flame sheets, which changes their total area and corresponding rate of reactant consumption. The total area of wrinkled laminar flamelets per unit volume is known as the flame surface density (FSD), and serves as the basis for a range of reduced order models for turbulent combustion [2,3]. It has been proposed [1], for example, that the turbulent burning velocity in this regime is related to the FSD by the relation

$$
\frac{S_{T}}{S_{L 0}}=I_{0} \int_{-\infty}^{\infty} \Sigma d \eta
$$

where $\mathrm{S}_{\mathrm{T}}$ and $\mathrm{S}_{\mathrm{L} 0}$ are the turbulent and laminar burning velocities, respectively, $\mathrm{I}_{0}$ is a stretch factor, $\Sigma$ is the flame surface density and $\eta$ is the direction normal to the mean flame-brush of a premixed turbulent flame. The physical mechanism relating $\mathrm{I}_{0}$ and $\Sigma$ in this regime is the flame-vortex interaction.

Extensive numerical and experimental studies have been made to characterize the physics of flame-vortex interaction with the aim of developing reduced order models to simulate it [1]. The response of premixed flames on curvature and strain has been investigated experimentally by isolated axisymmetric vortex rings $[4,5]$ interacting with laminar premixed flames. The advantage of these configurations is that they allow for repeatable flame-flowinteractions. Thus 2D measurement techniques at low repetition rates are sufficient to capture the full 3D character by phase dependent data acquisition. A realistic turbulent flow is not 
restricted to a single vortex size but contains a wide spectrum of eddies with the smaller ones randomly orientated. To explore interactions between turbulent flows and flame fronts measurements were also performed in turbulent premixed flames for example in flat flames stabilized in low-swirl burners [6], jet flames [7], V-flames [8], bluff-body stabilized flames [9], spherical flames in bombs [10,11] or free propagating spherical flames [12,13].

A key parameter of interest for FSD modeling is the local displacement velocity of the flamelet. The local displacement velocity is defined as the difference between the absolute velocity of the flamelet and the local convection velocity of the fluid. A key limitation of prior studies to measure this critical parameter is the inability of current generation planar imaging diagnostics such as particle image velocimetry (PIV) and planar laser-induced fluorescence (PLIF) to measure the three-dimensional orientation and displacement of a flamelet, or to track these parameters in time. Instead, efforts have focused mainly upon drawing conclusions about 3D displacement velocities from temporally unresolved, 2D measurements. Hartung et al. [9], for example, reports a sophisticated study wherein PIV and multi-shot OH-PLIF were applied simultaneously in a turbulent, bluff-body stabilized flame to measure 2D local flame displacement speed. DNS results on statistically planar, premixed turbulent flames [14] were later used to quantify the range of conditions wherein the probability density function (PDF) of measured 2D local flame displacement speed faithfully represents that of its 3D counterpart.

The aim of the present work is to directly measure the local 3D displacement velocity of a flame propagating through a flow with well-defined turbulence characteristics. This is accomplished through the use of a newly designed flow-facility that takes advantage of highspeed laser diagnostics to eliminate the need for flame stabilization. This paper presents both 
the burner, an overview of the diagnostics and data-reduction algorithm used to accomplish this and presents an initial statistical analysis of the measured data.

\section{Experimental facility}

Figure 1 shows the experimental set-up. The experiment consists of two components; a) a flow facility designed to produce a freely propagating flame in a flow with well-defined turbulence characteristics and b) a $10 \mathrm{kHz}$ frame-rate, dual-plane OH-PLIF and stereo-PIV measurement system. Each is described in detail below.

\subsection{Freely propagating flame facility}

The objective of measuring 3D local flame displacement velocity was achieved through the use of a new facility designed to produce unstabilized, freely propagating flames in a welldefined turbulent flow. This was accomplished by flowing a stoichiometric mixture of methane and air at low bulk velocity $(1.81 \mathrm{~m} / \mathrm{s})$ through a large diameter $(85 \mathrm{~mm})$ tube to produce a flow of Reynolds number 10.000. The tube was over 30 diameters in length, to ensure a fully-developed turbulent pipe flow velocity profile at the exit. Turbulence levels were $11 \%$ ???. The turbulent Reynolds number was determined to be 90 with the integral length scale estimated by the self-similarity assumption of a fully developed pipe flow [15]. The tube was surrounded by a $160 \mathrm{~mm}$ diameter, equivelocity co-flow of air in order to minimize shear and dilution by turbulent mixing with surrounding air. During an experiment, the flow of premixed stoichiometric methane-air was laser-ignited (1064 nm, $42 \mathrm{~mJ}$ pulse) at the tube exit. The resulting flame kernel grows and develops over the next $160 \mathrm{~mm}$ (ca. 2 tube diameters) into a wrinkled turbulent flame (see figure 2), which propagates through the measurement volume of dual-plane OH-PLIF/ stereo-PIV measurement system. After the flame passes the measurement volume the experiment is complete and methane to the facility is shut-off via a pneumatic valve. Each experiment run lasts approximately $40 \mathrm{~ms}$, during 
which 400 PLIF and 800 PIV measurements are captured, showing the period before, during and after the flame is present in the measurement volume. With loop recording, hundreds of facility firings could be realized during the measurement campaign.

\subsection{Diagnostics}

\section{Dual-plane OH-PLIF system}

Planar laser-induced fluorescence (PLIF) was used to image the combustion radical hydroxyl $(\mathrm{OH})$ in two parallel planes simultaneously at $10 \mathrm{kHz}$. As shown in Figure 1, this was accomplished using two independent OH-PLIF systems. Each system used a frequencydoubled dye laser (Sirah Credo, with Rhodamine 6G) pumped at $532 \mathrm{~nm}$ by a frequencydoubled, Q-switched diode-pumped solid state (DPSS) laser (Edgewave IS8II-E) to produce UV pulses of $0.15 \mathrm{~mJ}$ at $10 \mathrm{kHz}$. The lasers were tuned to $283.2 \mathrm{~nm}$ to excite the $\mathrm{Q}_{1}(7)$ line of the A-X (1-0) transition of $\mathrm{OH}$. Fluorescence emission at $310 \mathrm{~nm}$ was imaged onto CMOS cameras (LaVision HSS5, $512 \times 512$ pixels at $10 \mathrm{kHz}$ ) equipped with two-stage, lens-coupled intensifiers (LaVision, HS-IRO), UV-achromat lenses (Halle, f=150 mm, f/2.5) and hightransmission, bandpass interference filters (Laser Components UV-B). The field of view of each PLIF system was $14 \times 14 \mathrm{~mm}^{2}$. Flame luminosity and spontaneous $\mathrm{OH}^{*}$ chemiluminescence emission was supressed by gating the intensifier to 400 ns. Cross-talk between the systems was eliminated by offsetting image acquisition by $1 \mu$ s. Corrections for camera sensitivity and laser sheet profile non-uniformity were accomplished using standard correction techniques. The (380 $\mu \mathrm{m}$ thick) PLIF imaging planes were offset by $1 \mathrm{~mm}$ on either side of the PIV imaging plane. This separation distance was chosen to allow both an accurate interpolation of the flame position and flame-surface normal vector at the centerline (PIV) imaging plane. To avoid artificial smoothing of the measured displacement velocity, OH-PLIF was applied (separately) in the center plane to ensure that the radius of the smallest observed wrinkles was larger than the $2 \mathrm{~mm}$ plane separation of the PLIF system. 


\section{Stereoscopic particle image velocimetry system}

The 3-component velocity field was measured simultaneously using stereoscopic particle image velocimetry (SPIV). The SPIV system consists of a DPSS Nd:YVO $\mathrm{Y}_{4}$ laser (Edgewave IS4II-DE) and a pair of CMOS cameras (LaVision HSS6) equipped with $200 \mathrm{~mm}$ macro (Nikon AF-Nikkor, f/4) lenses and mounted 30 degrees off-normal with respect to the PLIF cameras. The laser and cameras were operated at $20 \mathrm{kHz}$ repetition rate, with interframe time set by the repetition rate (i.e. $50 \mu$ s). Scheimpflug adaptors were used to correct for off-axis defocussing. The flow was seeded with titanium-dioxide particles of nominal diameter $1 \mu \mathrm{m}$. The field of view of the system was $14 \times 14 \mathrm{~mm}^{2}$. Spatial calibration and de-warping of the PIV images were accomplished through the use of a three dimensional imaging target (Type 7, LaVision). Image cross-correlation and vector validation was performed with a multi-pass iterative cross-correlation algorithm (Davis 8, LaVision). Final interrogation window size and overlap were $16 \times 16$ pixels and $50 \%$ respectively. This corresponds to a vector spatial resolution of $0.44 \mathrm{~mm}$ and spacing of $0.22 \mathrm{~mm}$.

\section{Flame luminosity imaging}

A fifth camera was used to image the spark-ignition and subsequently the global flame luminosity during each run. This camera (LaVision HSS5), also operated at $10 \mathrm{kHz}$, was unintensified and was equipped with a $50 \mathrm{~mm}$, f/2.8 objective (Nikon, AF-Micro Nikkor). Figure 2 shows a sequence of flame luminosity images to give an impression of the global flame shape. This data was primarily used to verify the run-to-run repeatability of the laserignition system. 


\section{Data processing and local displacement speed reconstruction}

The absolute velocity of a flame ( $\vec{u}_{\text {flame }}$ ) is defined as the vector sum of its local displacement velocity $\left(\vec{u}_{d}\right)$ and the local fluid velocity $\left(\vec{u}_{\text {convection }}\right)$.

$$
\vec{u}_{\text {flame }}=\vec{u}_{d}+\vec{u}_{\text {convection }}
$$

The local displacement velocity $\vec{u}_{d}$ may be expressed as the displacement speed $\left(s_{d}\right)$ multiplied by the local flame-normal vector $(\vec{n})$

$$
\vec{u}_{d}=\vec{n} \cdot s_{d}
$$

The local displacement speed of the flame may then be expressed as

$$
\vec{n} \cdot S_{d}=\vec{u}_{\text {flame }}-\vec{u}_{\text {convection }}
$$

As the fluid velocity was measured in this study via SPIV, determination of the local displacement speed rests on an accurate determination of the total flame displacement and the orientation of the flame normal vector.

To determine the initial position and orientation of the flame, the reaction zones were identified in each pair of PLIF images. The images were first de-noised using a nonlinear diffusion filter [16] with anisotropic operator splitting. The flame fronts in the filtered images were then identified using a canny edge-detector. Once the reaction zones in each plane were identified, NURBS splines [17] were used to fit a three-dimensional surface between the discrete contours. Patch diffusion [18] was used to remove numerical noise from the fitted surface. This fitted surface was used to determine the flame position and orientation (flame surface normal) along the $\mathrm{Z}=0 \mathrm{~mm}$ plane, where the SPIV measurement is made. The result of one such contour fitting is shown schematically in Figure 3, together with the measured velocity field. 
With the center-plane position and orientation of the flame at time $\mathrm{t}$ now known, the simultaneously measured velocity field data is used to compute the position the contour would passively convect to at time $\mathrm{t}=\mathrm{t}+\Delta \mathrm{t}$. The remaining distance in the local flame normal direction to the second reconstructed instantaneous flame surface at $t+\Delta t$ is then a direct measure of the 3D local displacement speed for the period t to $t+\Delta t$.

The algorithm for determining local 3D displacement velocity was assessed using the generic test case shown schematically in Figure 4. The inclined red planes represent the flame surface as reconstructed from the dual OH-PLIF planes at times $t$ and $t+\Delta t$. Flame surface normal vectors of unity length are shown in the figure as red arrows. Displacement due to convection (as measured by the SPIV) is shown in blue. In this generic test case the planes (which represent the flame sheet) were convected at a constant velocity in axial direction only. The resulting local flame speed can be calculated analytically with the known input parameters (pixel displacement between planes, pixel displacement between two frames, flow velocity). An analysis on sensitivity of the precision reached on these input parameters was performed. The sensitivity of the algorithm to measurement uncertainty in the PIV data was also checked by adding artificial noise to the mean convection velocity field data in the generic test case. Starting from the flame in the central PIV-plane (blue thick line) convection alone transports the flame to the location marked by the green line. The local flame speed shown as red arrows result from the displacement between purely convected (virtual) and actual flame position. The precision was found to be better than 5\% assuming a laminar flame speed and $10 \%$ velocity noise independent from the angle of incidence.

As the algorithm for determining local 3D displacement velocity is based on separation of local fluid velocity from the absolute displacement of the flame, its accuracy and robustness 
are sensitive to the spatial location in the velocity field used to determine local convection velocity. If the convection velocity vector is taken from regions of elevated temperatures, as for example at the location of maximum $\mathrm{OH}$ gradients or even further in the burnt gas region, $S_{d}$ must be corrected for reduced gas densities [9]. It is also well known that the sudden change in seed-particle density across a flame front impacts the accuracy of the crosscorrelation algorithm used to determine velocity there, biasing the measurement toward regions of higher seed-particle density (i.e. the cold side). In addition, thermophoretic effects influence the accuracy of the PIV technique in the high temperature-gradient region of a flame [19]. For these reasons, the use of velocity data measured in the region of unburned reactants ahead of the propagating flame are the most appropriate choice for local convection velocity.

Based on laminar premixed flamelet calculations, the best compromise to obtain the local flame speed as close to the maximum heat release zone as possible while avoiding the discussed errors, was $0.4 \mathrm{~mm}$ upstream of the maximum $\mathrm{OH}$ gradient at the unburned gas side. From the flamelet calculations the maximum $\mathrm{OH}$ gradient, which was used to determine the flame front, was found to be $\sim 20 \mu \mathrm{m}$ on the unburned side of the position of maximum heat release. This prediction was verified through a sensitivity analysis, as shown in Figure 5. When using velocity vectors not $0.4 \mathrm{~mm}$ but $0.3 \mathrm{~mm}$ or $0.5 \mathrm{~mm}$ ahead of the flame front, the histogram of the local flame speed changes slightly, but the overall shape remains the same for all three cases. The mean value range from $0.77 \mathrm{~m} / \mathrm{s}$ for $0.3 \mathrm{~mm}$ offset to $0.52 \mathrm{~m} / \mathrm{s}$ for 0.5 $\mathrm{mm}(0.64 \mathrm{~m} / \mathrm{s}$ for $0.4 \mathrm{~mm})$.

\section{Results}

Figure 6 shows a time-sequence of measurements of a flame as it propagates through the flow, from right to left. The orientation of the flame surface, as determined from the dualplane OH-PLIF measurement is shown transecting the $\mathrm{Z}=0 \mathrm{~mm}$ plane. The flame-surface 
normal vectors along the $\mathrm{Z}=0 \mathrm{~mm}$ plane are shown in red. Also shown are the in-plane velocity vectors and 2D (out-of-plane) vorticity, as determined by the SPIV measurement. The sequence highlights the transient nature of the experiment, with the flame propagating freely through the measurement volume from one side to the other. The $10 \mathrm{kHz}$ repetition rate of the measurement system, combined with the $1.8 \mathrm{~m} / \mathrm{s}$ bulk velocity of the turbulent pipe flow results in exceptionally well-resolved time-series measurements of the interaction of the flame and the local flow field. Although the measurement volume spans only $10 \times 10 \mathrm{~mm}^{2}$, several tens of measurement frames can be acquired as the flame propagates through it. The velocity / vorticity fields shown in Figure 6 also demonstrate the high spatial resolution of the velocity field with respect to the scale of the flame-wrinkles under these conditions. Flame wrinkles are caused by the turbulent eddies interacting with the flame. The vorticity field reveals unorganized structures on the unburned side while flame-generated vorticity is observed on the burned side. [20] found that this flame generated vorticity and flame wrinkling is related to hydrodynamic instabilities resulting in tangential pressure gradients along the flame sheet.

Figure 7 shows 3 representative measurements of flame propagation. In this figure, the flame surface is rendered in red, the local fluid velocity in blue and the flame normal vectors in red. The magnitude of local 3D displacement velocity is represented by the colorbar of the flame contour along the $\mathrm{Z}=0 \mathrm{~mm}$ plane. The histograms on the right represent local displacement speed $s_{d}$ measured over a series of discrete intervals across the corresponding flame fronts. Figure 7 highlights several important phenomena. The flame in Figure 7 (top row)a shows a relatively low curvature in the $\mathrm{Z}=0 \mathrm{~mm}$ plane. The flame, however, is inclined $\approx 45^{\circ}$ with respect to the local fluid velocity. The corresponding histogram of $\mathrm{s}_{\mathrm{d}}$ is relative narrow $(\approx 0$ $0.8 \mathrm{~m} / \mathrm{s}$ ) and peaks close at $0.4 \mathrm{~m} / \mathrm{s}$, which is close to the laminar flame speed of the 
stoichiometric methane-air mixture. As expected, this is consistent with the laminar flamelet approximation. Figure 7 (middle row) shows a flame with greater in-plane curvature, and corresponding increase in the histogram width, suggesting flame wrinkling is beginning to have a measurable effect on the measured 3D local displacement speed. Figure 7 (bottom row) shows a flame with considerably higher in-plane curvature and a radically altered histogram of $s_{d}$. In this measurement we see high in-plane curvature toward the combustion products and corresponding increase in measured displacement velocity. In the region of lower-magnitude curvature (facing the unburned reactants), negative local displacement speeds are measured. These observations are consistent with the findings of [21]. Although a rigorous statistical analysis is beyond the scope of the present work, it is clear from Figure 7 that the measured data acquired in this study will allow a thorough analysis of the correlation between in-plane flame curvature and local 3D displacement speed. The highly resolved timeseries measurements captured by the $10 \mathrm{kHz}$ multi-plane OH-PLIF / SPIV diagnostics also enable one to track the orientation of the flame sheet with time and determine how it relates to local displacement speed.

Figure 8 shows a histogram of 3D local displacement speed measured during 49 test firings of the propagating flame facility. As the $10 \mathrm{kHz}$ acquisition rate of the PIV/PLIF measurement system results (deliberately, of course) in statistically non-independent (temporallycorrelated) measurements, only data from a single measurement frame of each test firing are included. The statistics are, nonetheless, well resolved with histogram comprising 12000 data points. The histogram in Figure 8 peaks at $0.34 \mathrm{~m} / \mathrm{s}$, very close to the laminar burning velocity $\mathrm{s}_{\mathrm{L}}$ of methane $(\approx 0.35 \mathrm{~m} / \mathrm{s}[22])$. The distribution, however, is quite broad, with a standard deviation of $\approx 1 \mathrm{~m} / \mathrm{s}$, or $\approx 2.5 \mathrm{~s}_{\mathrm{L}}$. The right (positive side) wing of the histogram is populated out to over $8 \mathrm{~s}_{\mathrm{L}}$. Such large deviations of $\mathrm{s}_{\mathrm{d}}$ from the unstretched laminar flame 
speed are uncommon (as shown by the low population of such values in the wings of Figure 8), but consistent with the findings of previous researchers [9,5].

The histogram in Figure 8 also shows a significant population of negative local 3D displacement speeds. This supports the finding of [21] who investigated two-dimensional stoichiometric methane flames using DNS and reported negative flame speeds in regions of high positive curvature due to diffusion effects in the tangential direction of the flame front. The physical mechanism responsible for these negative propagation velocities was not determined, but the data in Figure 8 show such values are statistically significant.

\section{Conclusions}

This paper describes an experimental study on the propagation of premixed flames through a flow with well-defined turbulence characteristics. The experimental facility, diagnostics and data analysis algorithms are described and initial results presented. Dual-plane OH-PLIF and SPIV were performed simultaneously at $10 \mathrm{kHz}$ to measure the local 3D displacement velocity of unstabilized, freely propagating flames passing through a premixed flow of methane and air. The experiment uses freely propagating flames to avoid spatiotemporal effects associated with burner- or aerodynamically-stabilized test flames. The sensitivity of the algorithm for computing local displacement velocity to various parameters was verified through a generic test flame case. Sample results showing the variation of local 3D displacement velocity vs. in-plane flame surface curvature and flame sheet orientation are presented. Statistics of local 3D displacement speeds are presented and show values ranging from slightly negative up to approximately eight times the laminar flame speed. 


\section{Acknowledgments}

The authors gratefully acknowledge financial support by the German Research Foundation

(DFG) through EXC 259. We would like to thank G. Künne for many inspiring discussions and are grateful to A. Ketelheun for performing the flamelet calculations. 


\section{References}

1. $\quad$ J.F. Driscoll, Prog. Energy Combust. Sci. (2008) 34, 91-134.

2. $\quad$ A.N. Lipatnikov, J. Chomiak, Prog. Energy Combust. Sci. (2002) 28, 1-74.

3. D. Veynante, L. Vervisch, Prog. Energy Combust. Sci. (2002) 28, 193-266.

4. C.J. Mueller, J.F. Driscoll, D.J. Sutkus, W.L. Roberts, M.C. Drake, M.D. Smooke, Combust. Flame 100 (1995), 323-331.

5. $\quad$ E.J. Long, G.K. Hargrave, Flow Turb. Combust. (2011) 86, 455-476.

6. I.G. Shepherd, R.K. Cheng, Combust. Flame (2001) 12, 2066-2075.

7. $\quad$ A.M. Steinberg, J. F. Driscoll, S.L. Ceccio, Exp. Fluids 44 (6) (2008), 985-999.

8. $\quad$ K.O. Smith, F.C. Gouldin. AIAA J 11 (5) (1979), 1243-1250.

9. G. Hartung, J. Hult, R. Balachandran, M.R. Mackley, C.F. Kaminski. Appl. Phys. B (2009) 96, 843-862.

10. H. Kido, M. Nakahara, K. Nakashima, J. Hashimoto, Proc. Combust. Inst. 29 ( 2002), 1855-1861.

11. D. Bradley, M.Z. Haq, R.A. Hicks, T. Kitagawa, M. Lawes, C.G.W. Sheppard, R. Woolley, Combust. Flame 133 (2003), 415-430.

12. B. Renou, A. Mura, E. Samson, A. Boukhalfa, Combust. Sci. Technol. (2002) 174:4, 143- 179.

21. T.-W. Lee, G.L. North, and D.A. Santavicca Combust. Flame 93 (1993), 445-456.

13. N. Chakraborty, G. Hartung, M. Katragadda, C.F. Kaminski, Combust. Flame (2011) 158, 1372-1390.

14. M. Desbrun, M. Mayer, P. Schröder, A.H. Barr, Proc. of the 26th annual conference on Computer graphics and interactive techniques (1999).

15. L. Piegl; Computer Graphics and Application, IEEE, Volume 11 Issue 1 (1991)

16. J. Weickert; Anisotropic Diffusion in Image Processing, Teubner Verlag, Stuttgart 1998.

17. C.J. Sung and C. K. Law, Combust. Sci. and Tech., 1994, Vol. 99, pp. 119-132

18. K.L. Pan, J. Qian, C.K. Law, W. Shyy, Proc. Combust. Inst. 29 (2002), 1695-1704.

19. I.R. Gran, T. Echekki, J.H. Chen, Proc. Combust. Inst. 26 (1996, 323-329.

20. T. Tahtouh, F. Halter, C. Mounaim-Rousselle, Combust. Flame 156 (2009), 17351743.

22. S.B. Pope, Turbulent flows, 2000, Cambridge, UK, Cambridge University press. 


\section{List of Figure Captions}

Figure 1: Experimental setup with cross section of burner and laser diagnostics system.

Figure 2: Temporal sequence of flame luminosity of the entire flame. Time between frames is $2 \mathrm{~ms}$.

Figure 3: Data from simultaneous dual-plane OH-PLIF and SPIV at one instant. In-plane velocities are denoted by arrows, the out-of-plane component is color-coded.

Figure 4: Generic test case to illustrate the determination of local flame displacement speed and assess precision; flame surface at time $t$ (red surface) and $t+\Delta t$ (red surface), displacement due to convection (blue arrow); flame displacement speed (red arrow), flame normal vectors (red arrow), absolute displacement (black arrow) and virtual flame location (green dots);

Figure 5: Histograms of local flame speed $s_{d}$ for different offset locations of flow velocities.

Figure 6: Time sequence of flame propagation (time between frames $100 \mu \mathrm{s}$ ). Out-of-plane vorticity is color-coded. The flame surface is shown as red plane. Its surface-normal vectors are shown as red arrows.

Figure 7: Instantaneous snapshot taken from different time sequences. Left: velocity vectors (blue arrows), flame surface (red) and its normal direction (red arrows). Color coded line indicates the local flame speed (m/s). Right: Corresponding single shot histograms

Figure 8: Histogram of local flame speeds extracted from 49 flame propagation events. 


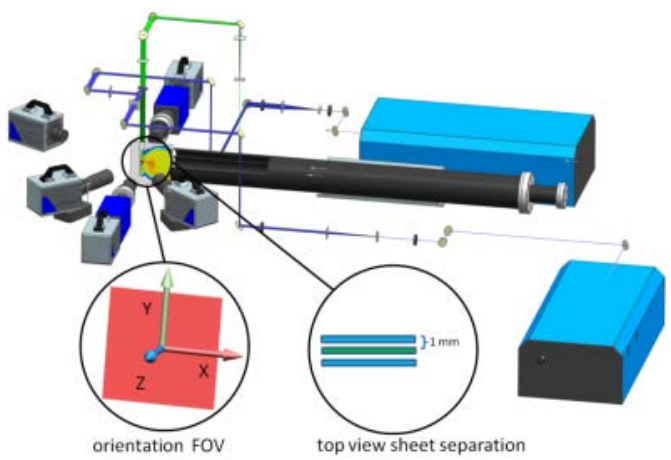

Figure 1: Experimental setup with cross section of burner and laser diagnostics system.

Single-column width (b/w) 


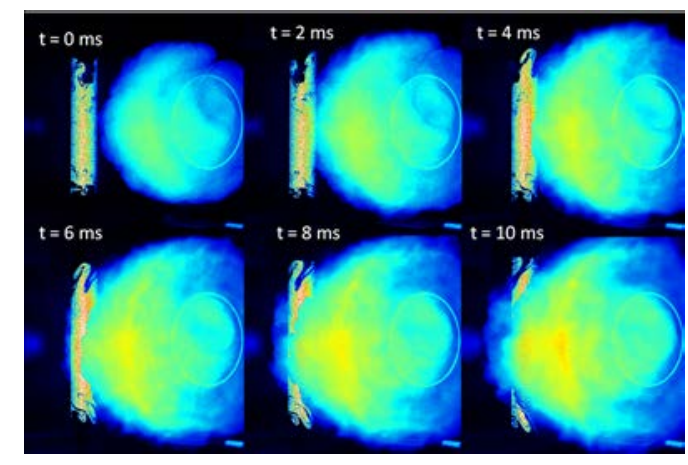

Figure 2: Temporal sequence of flame luminosity of the entire flame. Time between frames is $2 \mathrm{~ms}$.

Single-column width (b/w) 


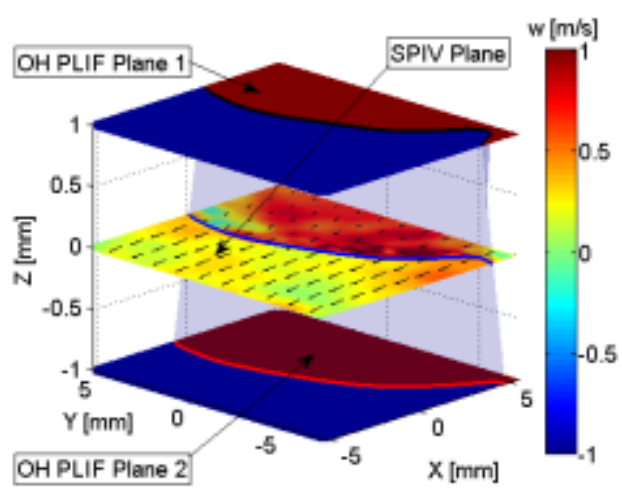

Figure 3: Data from simultaneous dual-plane OH-PLIF and SPIV at one instant. In-plane velocities are denoted by arrows, the out-of-plane component is color-coded.

Single-column width (color print) 


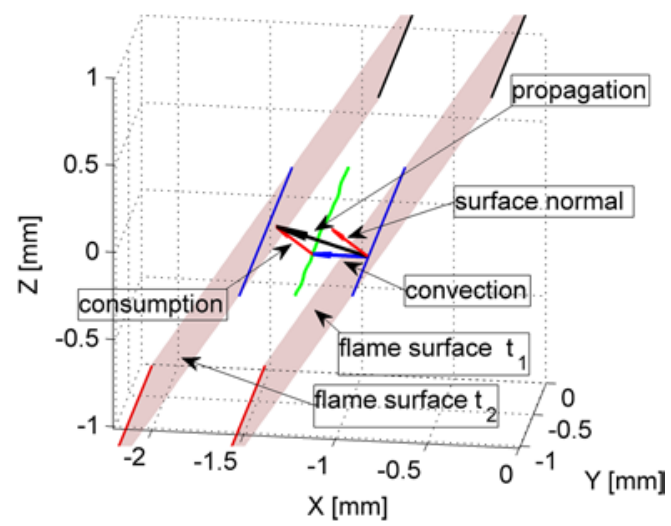

Figure 4: Generic test case to illustrate the determination of local flame displacement speed and assess precision; flame surface at time $\mathrm{t}$ (red surface) and $\mathrm{t}+\Delta \mathrm{t}$ (red surface), displacement due to convection (blue arrow); flame displacement speed (red arrow), flame normal vectors (red arrow), absolute displacement (black arrow) and virtual flame location (green dots);

Single-column width (color print) 


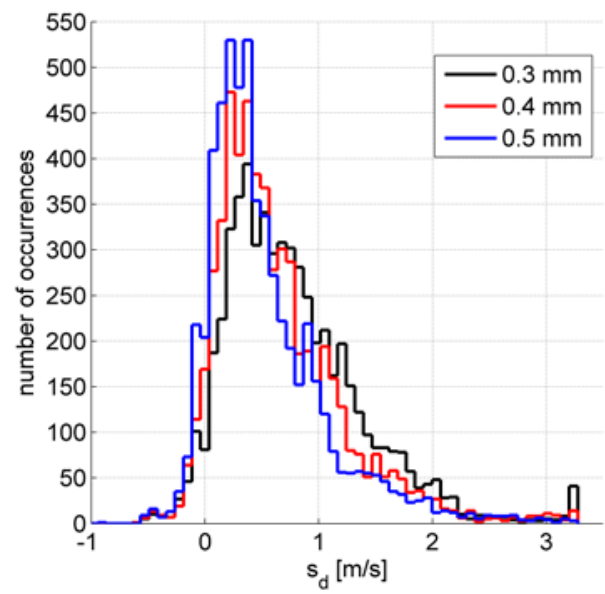

Figure 5: Histograms of local flame speed $s_{d}$ for different offset locations of flow velocities.

Single-column width (color print) 

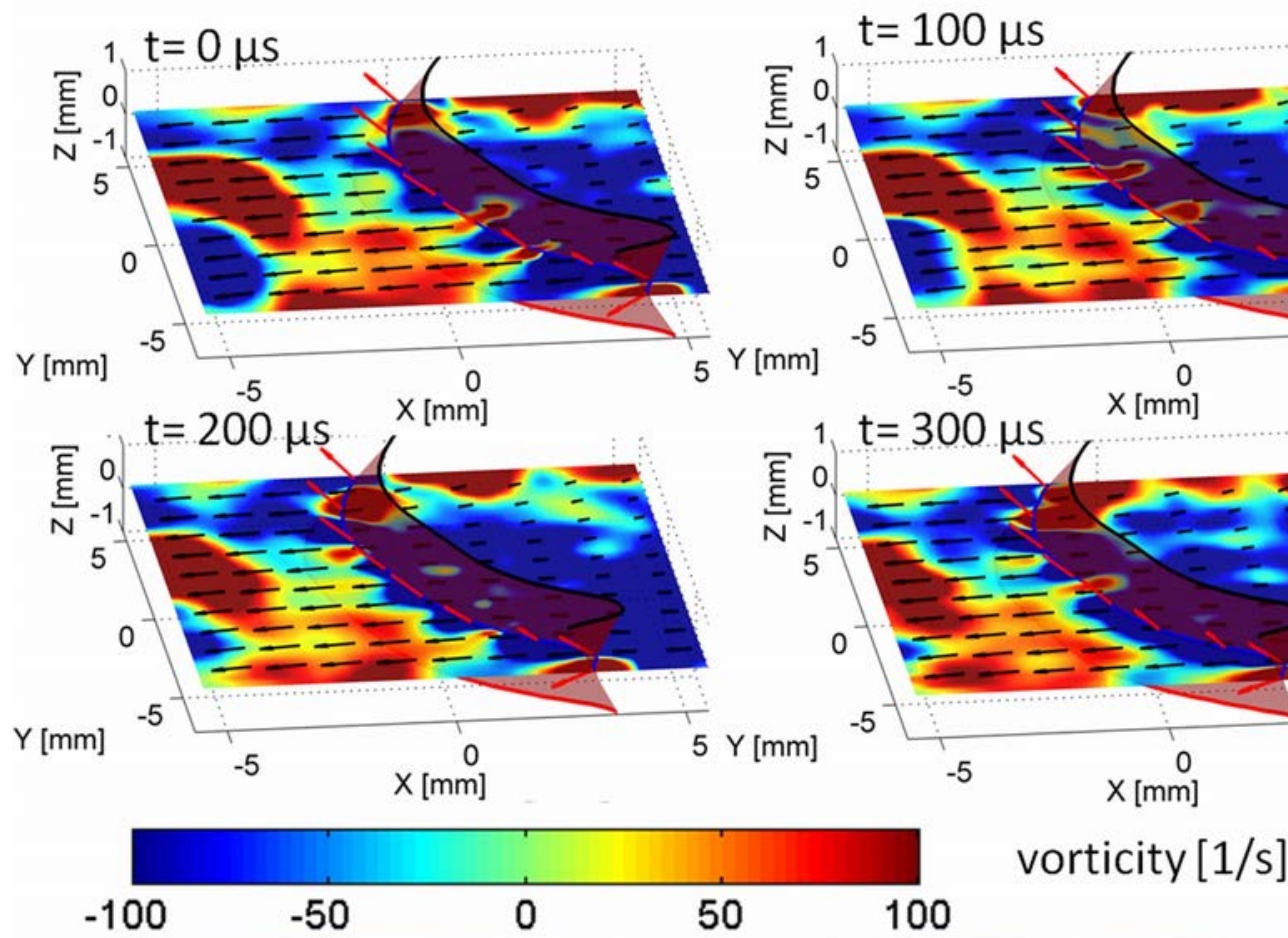

Figure 6: Time sequence of flame propagation (time between frames $100 \mu$ s). Out-of-plane vorticity is color-coded. The flame surface is shown as red plane. Its surface-normal vectors are shown as red arrows.

Double-column width (color print) 

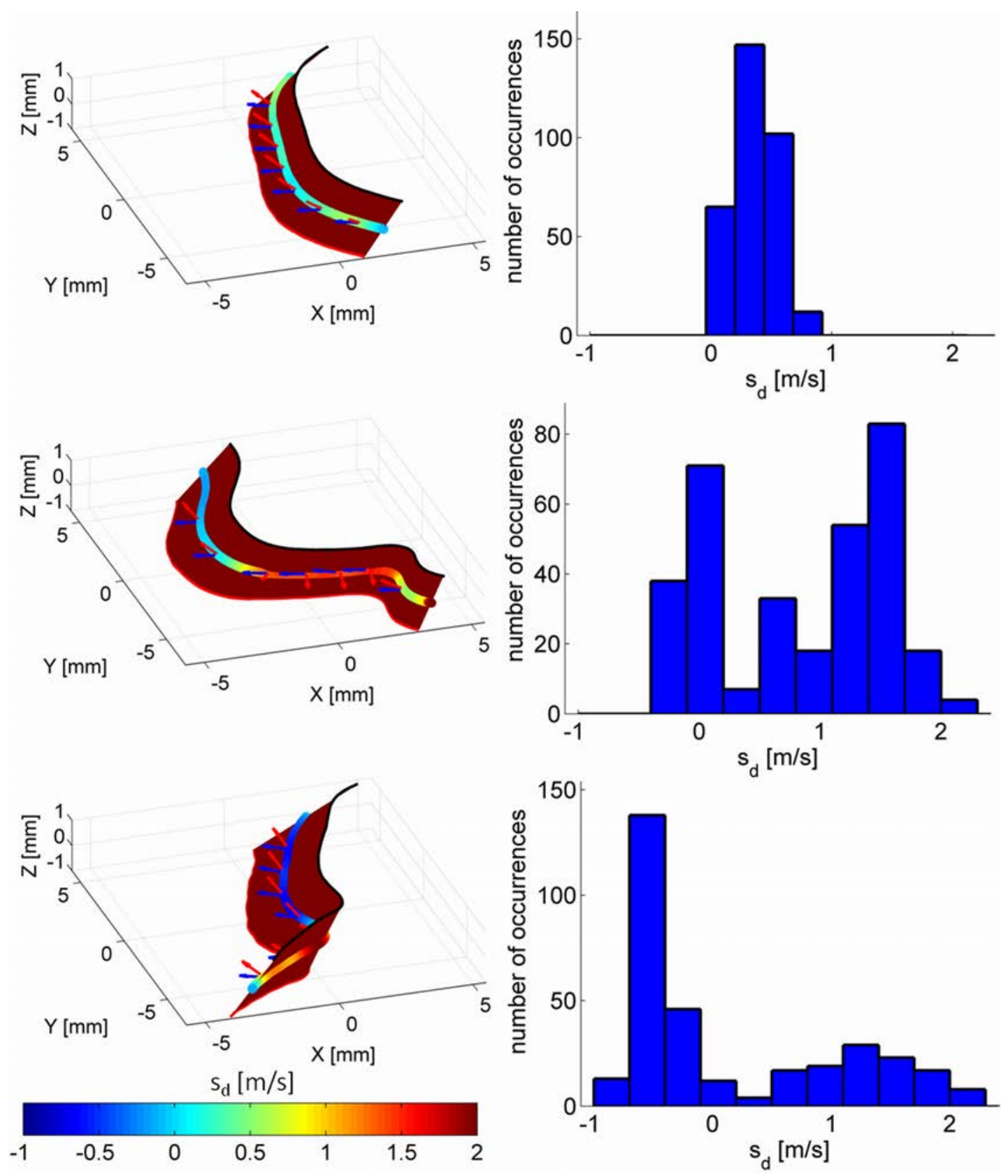

Figure 7: Instantaneous snapshot taken from different time sequences. Left: velocity vectors (blue arrows), flame surface (red) and its normal direction (red arrows). Color coded line indicates the local flame speed (m/s). Right: Corresponding single shot histograms

Double-column width (color print) 


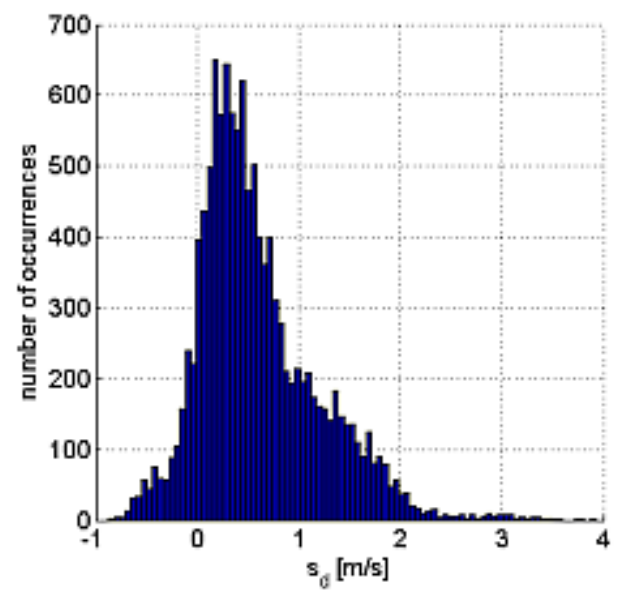

Figure 8: Histogram of local flame speeds extracted from 49 flame propagation events.

Single-column width (b/w) 